\title{
Theory of equidistant three-dimensional radiance measurements with optical microprobes
}

\author{
N. Fukshansky-Kazarinova, L. Fukshansky, M. Kühl, and B. B. Jørgensen
}

\begin{abstract}
Fiber-optic radiance microprobes, increasingly applied for measurements of internal light fields in living tissues, provide three-dimensional radiance distribution solids and radiant energy fluence rates at different depths of turbid samples. These data are, however, distorted because of an inherent feature of optical fibers: nonuniform angular sensitivity. Because of this property a radiance microprobe during a single measurement partly underestimates light from the envisaged direction and partly senses light from other directions. A theory of three-dimensional equidistant radiance measurements has been developed that provides correction for this instrumental error using the independently obtained function of the angular sensitivity of the microprobe. For the first time, as far as we know, the measurements performed with different radiance microprobes are comparable. An example of application is presented. The limitations of this theory and the prospects for this approach are discussed.

Key words: Optics of living tissue, radiance measurements, fiber-optic microprobe, threedimensional picture of light field in turbid media. 수 1996 Optical Society of America
\end{abstract}

\section{Introduction}

Direct measurements of radiation fields in absorbing turbid media with optical fibers (microprobes) have been increasingly employed in the past few years, especially to study the optics of living tissues, ${ }^{1-4}$ aquatic sediments, and microbial mats (ecosystems located on sediments in shallow water and consisting of dense populations of microalgae and bacteria). ${ }^{5-8}$ The purposes of these measurements are:

(1) To find the spectral light fluxes and the angular distributions of radiance at different depths of a sample for a given light source and experimental setup (evaluation of light field parameters).

(2) To calculate the optical properties of the tissue or sediment (optical cross sections and phase functions) on the basis of a sufficient set of measured light field parameters (elucidation of the inherent optical properties).

N. Fukshansky-Kazarinova, M. Kühl, and B. B. Jørgensen are with the Max-Planck-Institute of Marine Microbiology, Fahrenheitstrasse 1, D-28359 Bremen, Germany. L. Fukshansky is with the Institute of Biology 2, University of Freiburg, Schänzlestrasse 1, D-79104 Freiburg, Germany.

Received 10 February 1995; revised manuscript received 5 July 1995.

0003-6935/96/010065-09\$06.00/0

(c) 1996 Optical Society of America
The first step reveals the light microenvironment at any point on an individual sample and permits a comparison between the spatial pattern of light and those of the light-driven processes, e.g., photosynthesis and changes in the structure of a microbial ecosystem. The second step opens the way for modeling and general analysis of the light-driven processes without limitations imposed by the specific features of individual samples.

\section{A. Optical Microprobes and Geometry of Measurements}

Three basic types of optical fiber probe exist. The radiance microprobes with a tip diameter as small as $10 \mu \mathrm{m}$ sense the incoming radiation within the solid angle of approximately $10-20^{\circ}$ around their axis. The fluence rate optical probes, also called scalar irradiance probes, with a tip diameter as small as 40 $\mu \mathrm{m}$ sense the total radiant flux from all directions and thus provide the fluence rate. The irradiance probes measure the hemispheric radiant flux incident from the hemisphere, which is oriented normal to the axis of the probe; these probes have the same size as the fluence rate probes and are in fact small cosine collectors. Among these types the radiance microprobe is the only tool that provides the angular distribution of radiance. Furthermore, owing to its size, it is most appropriate for studies on a small geometric scale, in particular within thin samples. 
It is this type of optical microprobe that we address in this paper.

Radiance microprobes have well-defined lightcollecting properties and ensure radiance measurements with high spatial resolution at different depths and in different directions. The directional sensitivity of a radiance microprobe (Fig. 1 ) is specified by a numerical aperture of $n_{0} \sin \left(\theta_{a}\right)$, where $n_{0}$ is the refractive index of the medium and $\theta_{a}$ is the acceptance half-angle of the optical fiber. ${ }^{9}$ Radiance fiber probes thus have a larger acceptance half-angle in air $\left(n_{0}=1\right)$ than in water $\left(n_{0}=1.33\right)$. For technical details, preparation, and tests see Refs. 2, 5, and 6 .

The following experimental setup is generally used. The input end of a microprobe can be advanced to any required depth and in any required direction into a plane-parallel sample (leaf, sediment) by means of a micromanipulator. The output end is connected to the spectral detector system based on a sensitive diode array (for more details refer to Ref. 6). The light field in a sample is assumed to exhibit axial symmetry around the vertical incident, collimated light. Each radiance measurement at zenith angle $\theta_{i}(i=0,1, \ldots)$ relative to the light source covers an angular interval of $2 \alpha$ around the direction of measurement (Fig. 2). Such a measurement is assumed to be representative for the $i$ th spherical band having a width of $2 \alpha$. The entire unit sphere of radiance directions appears subdivided into $K$ nonoverlapping spherical bands with a zenithal size of $2 \alpha$; the radiance within a band is assumed to have a constant value $L_{i}$. Throughout this paper we use the designation spherical band instead of the rigorous but somewhat lengthy surface of the spherical layer. The notion is, however, transparent and clearly specified in Fig. 2 . As seen in Fig. 2 each measurement at $\theta_{i}(i=1,2 \ldots)$ covers a zenith angle of $2 \alpha$, the measurement at $\theta_{0}$ covers zenith angle $\alpha$, the measurement in the opposite $\theta_{0}$ direction (which

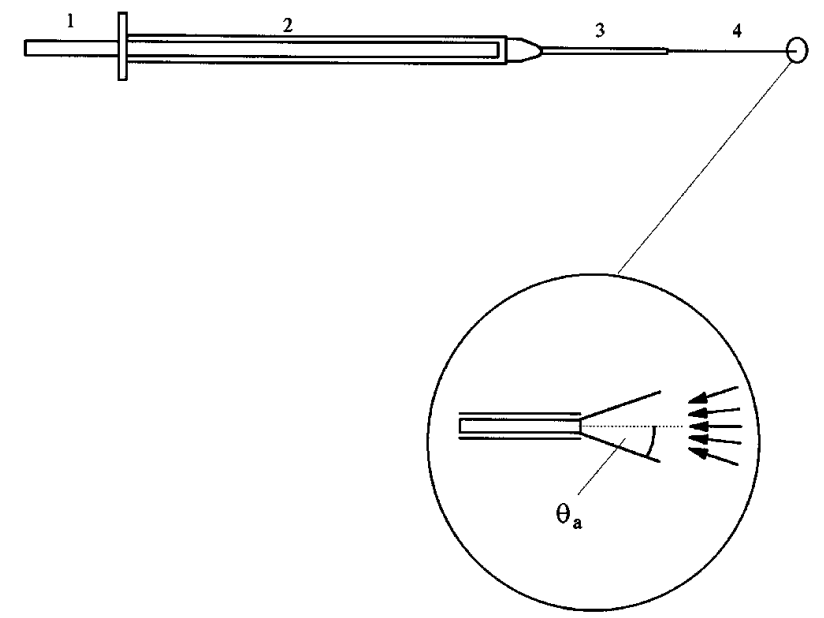

Fig. 1. Fiber-optic radiance microprobe: 1, fiber cable; 2, syringe; 3 , hypodermic needle; 4 , optical fiber. $\theta_{a}$ is the apex angle of the circular cone of collection also known as the acceptance angle of the probe.

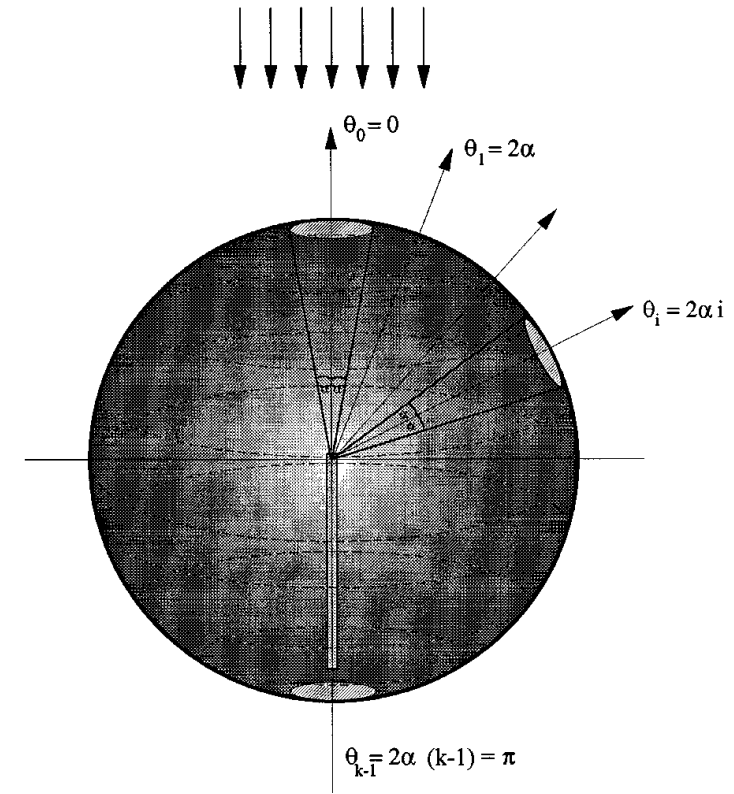

Fig. 2. Spatial design of three-dimensional radiance measurements: $\theta_{0}=0, \theta_{1}, \ldots \theta_{i}, \ldots \theta_{k-1}=\pi$ represents the direction of measurements. Each measurement represents the radiance within the corresponding spherical band. The radiance value within a band is assumed to be constant.

would cover zenith angle $\alpha$ ) is omitted since it interferes with the incident light. Therefore, the number of bands is related to angle $\alpha$ as

$$
K=\frac{\pi}{2 \alpha}+1
$$

with the number of measurements being $K-1$. Thus we have $K-1$ measured quantities $M_{i}(i=0$, $\ldots, K-2)$ and $K$ unknown quantities $L_{i}(i=0, \ldots$, $K-1)$ to be derived from the $M_{i}$ 's. To have a determined solvable problem the quantity $M_{k-1}$, which is not observable for technical reasons (see above), should be extrapolated on the basis of other $M_{i}$ 's. Figure 2 represents the case of $\alpha=10^{\circ} ; K=10$.

The experimental setup described above corresponds to the theoretical model of light propagation known as multiflux approximation of the equation of radiative transfer. ${ }^{10}$ A measurement in direction $\theta_{i}$ is assumed to give radiance $L_{i}$ in any direction $\theta$ within the solid angle that corresponds to band $i$, the value $L_{i}$ being constant for all directions $\theta$ within band $i$. In addition, any flux at this point can be calculated on the basis of $L_{i}$ values and the known relationship between the surfaces of spherical bands. The radiant energy fluence rate is simply the sum of $L_{i}$ 's weighted with the fractional areas of the corresponding spherical bands on the unit sphere.

\section{B. Instrumental Error and the Problem of Its Correction}

However, the basic assumption of this measurement scheme, i.e., that each individual measurement yields the true value of $L_{i}$, is not fulfilled. For a microprobe 
to perceive the real $L_{i}$ value, it should have an acceptance half-angle $\theta_{a}$ equal to $\alpha$, the half-angle of the spherical band chosen. It is not warranted that a microprobe with $\theta_{a}=\alpha$, where $\alpha$ obeys Eq. (1) for integer $K$, will always be disposable. Even more important is that an ideal microprobe should have a uniform angular sensitivity within the angular area restricted to the measured band (i.e., within $\theta_{a}=\alpha$ ) and zero sensitivity outside this area. Such ideal sensitivity is presented as the dashed line in Fig. 3 and is never the case. The real angular sensitivity is a continuous bell-shaped surface $h(\vartheta)$, where $\vartheta$ is the angular deviation from the axis of the microprobe in the local coordinate system associated with the microprobe. The continuous curve $h(\vartheta)$ in Fig. 3 is a projection of this surface onto a vertical plane that contains the incident light direction. Obviously, the real, measured quantity $M_{i}$ will deviate from $L_{i}$ since it underestimates the radiance within band $i$ and includes superfluous contributions at least from the neighboring bands $i-1$ and $i+1$ (shaded area in Fig. 3).

The prerequisite for proper use of the radiance microprobe measurements, especially for estimation of the inherent optical parameters, is a theory which, for a given angular sensitivity of probe $h(\vartheta)$ and spherical bandwidth $2 \alpha$, can be used to obtain the true values of radiance $L_{i}$ on the basis of a set of the measured quantities $M_{i}$. The aim of our paper is to develop such a theory. We present a general approach and correcting formulas for the case in which only the effects of the neighboring bands are accounted for. In Subsection 2.A we formulate the problem in mathematical terms and derive a general relationship between the measured quantities and radiances. This relationship contains complicated

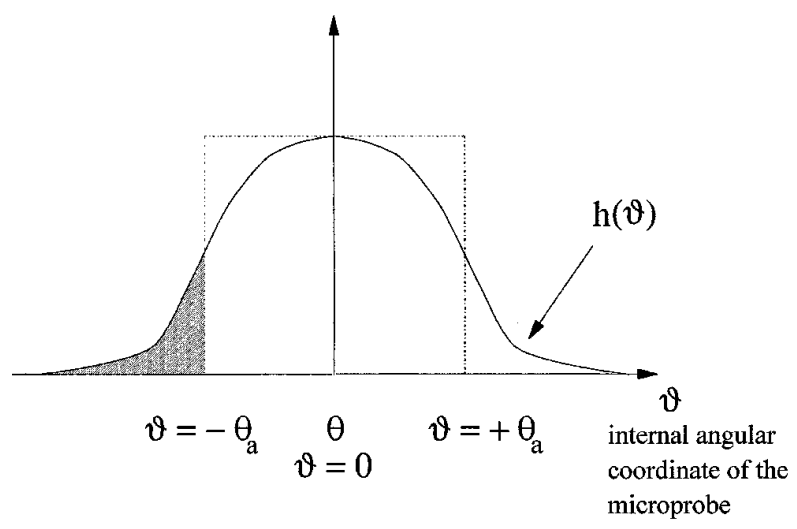

Fig. 3. Angular sensitivity distribution of a microprobe oriented in the $\theta$ direction: $\vartheta$ is the internal angular coordinate, $\vartheta=0$ coincides with $\theta, h(\vartheta)$ is the relative sensitivity, $h(\vartheta)$ is maximal in the direction of the microprobe axis $(\vartheta=0)$ whereas light that deviates from $\vartheta=0$ is captured with lower sensitivity. Moreover, the probe senses light from neighboring spheric bands (at $\vartheta>+\theta_{a}$ and $\left.\vartheta<-\theta_{a}\right)$. The dashed line shows the ideal angular sensitivity that is required to ensure that each measurement provides an undistorted value of the constant radiance within the corresponding spherical band. surface integrals. We develop an auxiliary construction that facilitates the solution of these integrals in Subsection 2.B. The final solution of the problem is presented in Subsection 2.C. An example of real data processing based on this theory is given in Subsection 2.D. Section 3 contains a short discussion.

\section{Results}

\section{A. Mathematical Formulation of the Problem}

We consider radiation that propagates in a planeparallel horizontal sample, in which the collimated incident light is directed vertically downward. The angular distribution of radiance at some point $\mathrm{P}$ depends only on its depth. Introducing spherical coordinates $\theta$ (zenith angle) and $\varphi$ (azimuth angle) associated with a unit sphere circumscribing point $P$, we assume that the radiance at any point $(\theta, \varphi)$ depends only on $\theta$, but not on $\varphi$ (azimuthal symmetry). To estimate the angular radiance distribution at point $\mathrm{P}$, we applied the optical microprobe shown in Fig. 2. The measurements begin with $\theta_{0}=0$, i.e., in the direction of the source, and proceed with step $2 \alpha$ until point $\theta=\pi$. The results of these measurements are designated $M_{i}(i=0,1, \ldots, K-1)$. The zenithal distribution of radiance $f(\theta)$ is assumed to be a stepwise constant function with constant values $L_{i}$ within spherical bands $\left(\theta_{i}-\alpha \leq \theta \leq \theta_{i}+\alpha\right.$, $0 \leq \varphi \leq 2 \pi$ ).

The problem is to estimate $L_{i}$ on the basis of the measured quantities $M_{i}(i=0,1, \ldots, K-1)$. We consider an individual measurement under zenith angle $\theta=\theta_{i}$ and introduce local Cartesian coordinates $x, y, z$ as well as corresponding spherical coordinates $\vartheta, \psi$ associated with point $\mathrm{P}$ and direction $\theta_{i}$, so that $\vartheta=0$ when $\theta=\theta_{i}$ (see Fig. 4). If the probe captures only the radiation that is incident on the spherical surface, $S_{0}$ : $(0 \leq \vartheta \leq \alpha, 0 \leq \psi \leq 2 \pi)$, and this radiation is sensed without loss (i.e., with $100 \%$ sensitivity), then the connection between $M_{i}$ and $L_{i}$ appears trivial:

$$
M_{i}=\iint_{S_{0}} f(\vartheta) h(\vartheta) \mathrm{d} S=L_{i} S_{0}
$$

where $h(\vartheta)=1$ is the constant ideal angular sensitivity of the microprobe and $f(\vartheta)=L_{i}$ is the constant radiance within band $i$.

However, as described in Section 1, the bell-shaped function $h(\vartheta)=1$ only for $\vartheta=0$ and is a decreasing function for $\vartheta>0$. Furthermore, radiation that enters two neighboring spherical bands also contributes to the value of $M_{i}$. In fact, the measured radiation is spread over the spherical surface $S$ : $(0 \leq \vartheta \leq 3 \alpha, 0 \leq \psi \leq 2 \pi)$, which consists of three parts, $S_{i i-1}, S_{i i}, S_{i i+1}$, belonging to three different spherical bands with constant radiance values of 


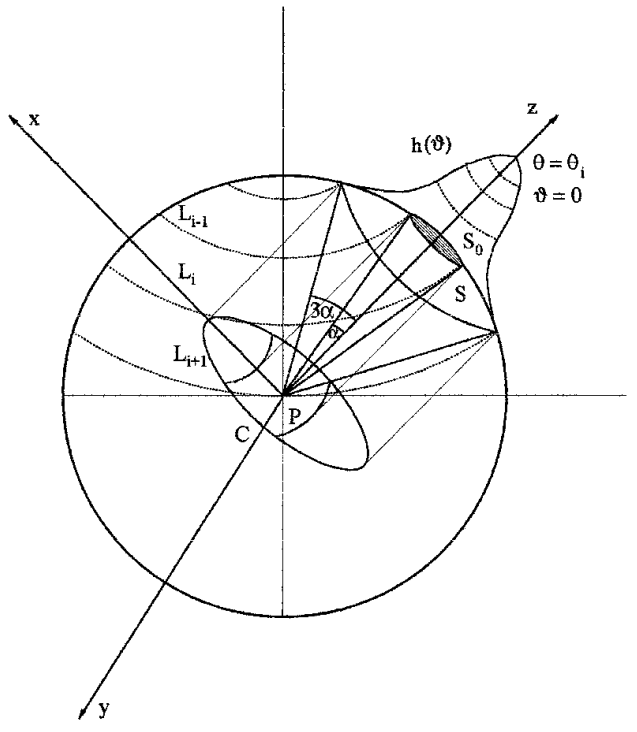

Fig. 4. Spatial and sensitivity relations between different components of radiation sensed in the course of a single measurement at point $\mathrm{P}$ under zenith angle $\theta=\theta_{i} . \quad x, y$, and $z$ are local Cartesian coordinates and $\vartheta$ is the local spherical coordinate associated with this measurement. $\vartheta$ is zero in the $\theta_{i}$ direction. $h(\vartheta)$ is the angular sensitivity of the microprobe. $L_{i-1}, L_{i}$, and $L_{i+1}$ are the unknown radiances within spherical bands $i-1, i, i+1$, respectively. $\alpha$ is the half-angle of the zenithal angular interval that corresponds to a single measurement; thus $\alpha$ is the apex angle of the circular cone whose cross section with the unit sphere-spherical surface $S_{0}$-matches the zenithal angular interval of the measured spherical band (in this case band number i). The entire radiation sensed in the course of this measurement is spread over spherical surface $S$, which includes parts of spherical bands $i-1, i, i+1$ and is the cross section of the unit sphere with a circular cone having apex angle $3 \alpha$. Circle $C$ is a mapping of spherical surface $S$ onto the $x P y$ plane.

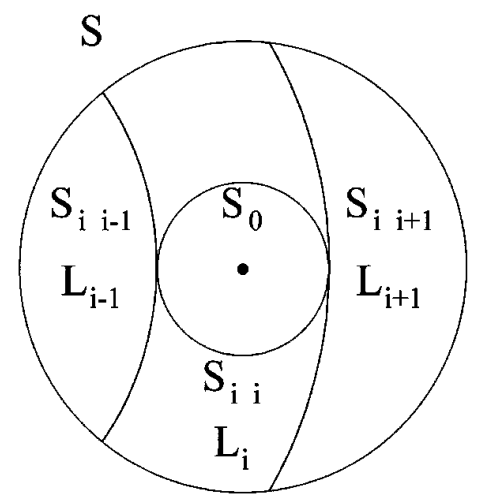

Fig. 5. Structure of sphercial surface $S$, which perceives the entire radiation sensed in measurement number $i . \quad S_{i i-1}, S_{i i}$, and $S_{i i+1}$ are parts of $S$ that belong to sphercial bands $i-1, i, i+1$ and, therefore, perceive radiances $L_{i-1}, L_{1}, L_{i+1}$, respectively. $S_{0} \subset$ $S_{i i}$ is the spherical surface that would perceive the entire radiation sensed in measurement number $i$ if the angular sensitivity of the microprobe had an ideal rectangular shape with acceptance angle $\alpha$ as presented by the dashed line in Fig. 3. For the position of surface $S$ on the unit sphere see Fig. 4.

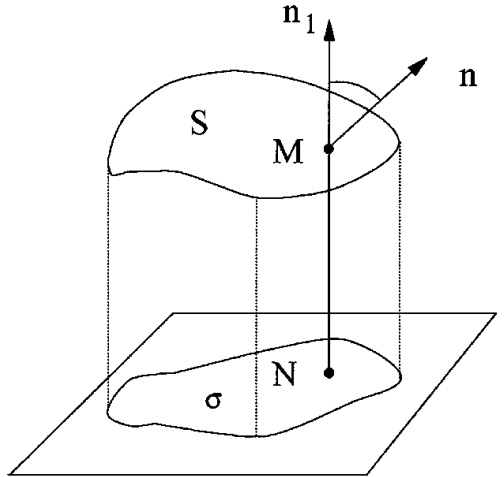

Fig. 6. Illustration of the transition from the integral over a curvilinear surface $S$ to the integral over a plane surface $\sigma . \quad n$ and $n_{1}$ are the normals to surfaces $S$ and $\sigma$, respectively. $\quad M$ and $N$ are the current points on surfaces $S$ and $\sigma$, respectively.

$L_{i-1}, L_{i}, L_{i+1}$, respectively (Fig. 5). Thus

$$
\begin{aligned}
M_{i}= & \iint_{S} f(\vartheta) h(\vartheta) \mathrm{d} S \\
= & L_{i-1} \iint_{S_{i i-1}} h(\vartheta) \mathrm{d} S+L_{i} \iint_{S_{i i}} h(\vartheta) \mathrm{d} S \\
& +L_{i+1} \iint_{S_{i i+1}} h(\vartheta) \mathrm{d} S \\
= & L_{i-1} J_{i i-1}+L_{i} J_{i i}+L_{i+1} J_{i i+1} .
\end{aligned}
$$

An integral of type $J_{i j}$ over the curvilinear surface can be expressed by an integral over a plane area and then by a standard repeated integral. Because of the complex geometry, the calculation of integrals $J_{i j}$ requires a major effort. In Subsection 2.B we provide an auxiliary construction to facilitate this calculation.

\section{B. Facilitating the Calculation of Integrals $J_{i j}$}

Let $S$ be an area on a curvilinear surface, $\sigma$ its projection on a plane, and $\left(n, n_{1}\right)$ the angle between the normal to $S$ at point $\mathrm{M}$ and the normal to the plane (Fig. 6). Then

$$
\iint_{S} f(M) \mathrm{d} S=\iint_{\sigma} \frac{f(N)}{\cos \left(n, n_{1}\right)} \mathrm{d} \sigma
$$

where $N$ is the projection of point $M$ onto the plane and $f(M)=f(N)$. To calculate the integrals in Eq. (3) we map spherical surface $S$ onto the $x P y$ plane (see Fig. 4). In this case

$\left(n, n_{1}\right)=\vartheta$

the circle surrounding area $S$ is transformed without any distortion in circle $C$, with the center at point $\mathrm{P}$ and radius $R=\sin (3 \alpha)$;

the circles delimiting the areas $S_{i i-1}, S_{i i}, S_{i i+1}$ (refer to Fig. 5) are transformed into ellipses designated as $C_{i i-1}$ and $C_{i i+1}$ in Fig. 7. 


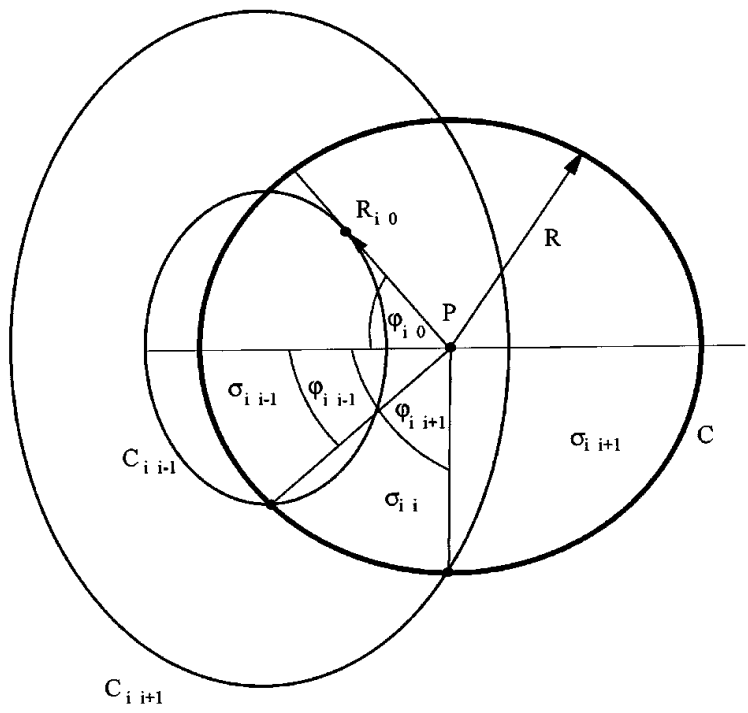

Fig. 7. Mapping of the surface of a unit sphere onto the $x P y$ plane that is normal to the $\theta_{i}$ direction of measurement number $i$ (see Fig. 4). Circle $C$ with diameter $R=\sin (3 \alpha)$ is the mapping of spherical surface $S$. Ellipses $C_{i i-1}$ and $C_{i i+1}$ are mappings of circles that delimit the spherical bands $i-1, i, i+1$ and, in particular, areas $S_{i i-1}, S_{i}$, and $S_{i i+1}$ inside surface $S$, respectively (see Fig. 5). Areas $\sigma_{i i-1}, \sigma_{i}$, and $\sigma_{i i+1}$ are mappings of spherical surfaces $S_{i i-1}, S_{i}$, and $S_{i i+1}$, respectively. $\varphi_{i i \pm 1}$ and $R$ are polar coordinates of points of intersection of ellipses $C_{i i \pm 1}$ with circle C. $\varphi_{i 0}$ and $R_{i 0}$ are polar coordinates of the point of contact of the tangent to ellipse $C_{i i-1}$ from point $\mathrm{P}$.

Let us designate the half-axes of ellipses $C_{i i \pm 1}$ along the $x$ and $y$ axes as $a_{i i \pm 1}$ and $b_{i i \pm 1}$, respectively, and the distance of the centers of ellipses from point $\mathrm{P}$ as $q_{i i \pm 1}$. One can see that

$$
\begin{aligned}
a_{i i \pm 1} & =\sin \left(\theta_{i} \pm \alpha\right) \cos \left(\theta_{i}\right), \\
b_{i i \pm 1} & =\sin \left(\theta_{i} \pm \alpha\right), \\
q_{i i \pm 1} & =\cos \left(\theta_{i} \pm \alpha\right) \sin \left(\theta_{i}\right), \\
a_{0,-1} & =b_{0,-1}=a_{k-1, k}=b_{k-1, k}=0 .
\end{aligned}
$$

Now we introduce on the $x P y$ plane polar coordinates $r, \varphi$. For these coordinates the equations for the projections of spherical surfaces on the $x P y$ plane are

for $C, r=R[R=\sin (3 \alpha)$ as described above $]$;

for $C_{i i \pm 1}, r=f_{i i \pm 1}(\varphi)$.

We designate the values of coordinate $\varphi$ at which ellipses $C_{i i \pm 1}$ cross circle $C$ as $\varphi_{i i \pm 1}$ (refer to Fig. 7). Since in our set of measurements $\theta_{i}$ is symmetrical with respect to point $\theta=\pi / 2$, we can confine our consideration to the subset $\theta<\pi / 2$ (in the case of $\theta=\pi / 2$ both ellipses $C_{i i \pm 1}$ degenerate into straight lines). Note that point $\mathrm{P}$ is always located inside ellipse $C_{i i+1}$ and outside ellipse $C_{i i-1}$. This means that one can always draw a tangent to ellipse $C_{i i-1}$ from point $\mathrm{P}$; coordinates of the point of contact are designated $\left(R_{i 0}, \varphi_{i 0}\right)$. Equation $r=f_{i i-1}(\varphi)$ assigns to

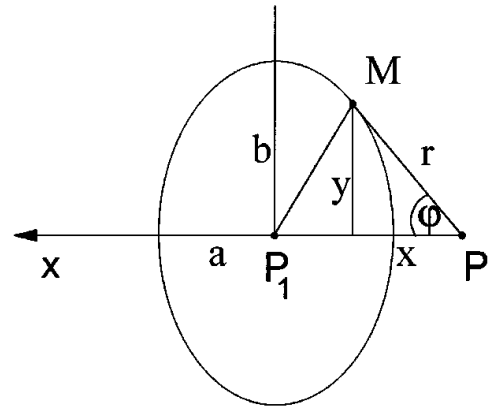

Fig. 8. Derivation of the parametric description of ellipses $C_{i i \pm 1}$ presented in Fig. 7 and given in Appendix A. $x$ and $y$ are Cartesian coordinates, and $\varphi$ and $r$ are polar coordinates on the $x P y$ plane. $a$ and $b$ are the axes of the ellipse with center $P_{1} . \quad M$ is the point of contact of the tangent to the ellipse from point $\mathrm{P}$, which is outside the ellipse because $P_{1} P=q>a$.

each value $0 \leq \varphi \leq \varphi_{i 0}$ two values of $r$, i.e., $f_{i i-1}(\varphi)$ is a two-digit function.

We now show the functions $f_{i i \pm 1}(\varphi)$ and other parameters defined above as being expressed through the parameters of ellipses listed in Eqs. (5) (for the derivation see Fig. 8 and Appendix A). We designate

$$
\begin{aligned}
f_{i j}{ }^{ \pm}(\varphi)= & \frac{b_{i j}}{a_{i j}{ }^{2}+c_{i j}{ }^{2} \cos ^{2}(\varphi)}\left\{q_{i j} b_{i j} \cos (\varphi)\right. \\
& \left. \pm a_{i j}\left[a_{i j}{ }^{2}-{q_{i j}}^{2}+\left({q_{i j}}^{2}+c_{i j}{ }^{2}\right) \cos ^{2}(\varphi)\right]^{1 / 2}\right\}, \\
g_{i j}= & \frac{1}{R c_{i j}{ }^{2}}\left\{q_{i j}{ }^{2} b_{i j}{ }^{2}-a_{i j}{ }^{2}\right. \\
& \left.\times\left[q_{i j}{ }^{2} b_{i j}{ }^{2}-c_{i j}{ }^{2}\left(R^{2}-b_{i j}{ }^{2}\right)\right]^{1 / 2}\right\},
\end{aligned}
$$

where $c_{i j}{ }^{2}=b_{i j}{ }^{2}-a_{i j}{ }^{2}$. Then for $C_{i i-1}$,

$$
\begin{aligned}
r & =f_{i i-1}{ }^{ \pm}(\varphi), \\
\cos \left(\varphi_{i i-1}\right) & =g_{i i-1}, \\
\cos \left(\varphi_{i 0}\right) & =\left(\frac{q_{i i-1}{ }^{2}-{a_{i i-1}}^{2}}{q_{i i-1}{ }^{2}+c_{i i-1}^{2}}\right)^{1 / 2}, \\
R_{i 0} & =\frac{1}{q_{i i-1}}\left[\left(q_{i i-1}^{2}-{a_{i i-1}}^{2}\right)\left(q_{i i-1}^{2}-c_{i i-1}{ }^{2}\right)\right]^{1 / 2},
\end{aligned}
$$

and for $C_{i i+1}$,

$$
\begin{aligned}
r & =f_{i i+1}{ }^{+}(\varphi), \\
\cos \left(\varphi_{i i+1}\right) & =g_{i i+1} .
\end{aligned}
$$

C. Calculating Integrals $J_{i j}$

Now we can calculate the integrals given in Eq. (3). When we calculate the integral

$$
J_{i i-1}=\iint_{\sigma_{i i-1}} \frac{h(\vartheta)}{\cos (\vartheta)} \mathrm{d} \sigma
$$

three cases can occur: 
(1) $R_{i 0} \geq R$, i.e., the point of contact of ellipse $C_{i i-1}$ with the tangent drawn through point $\mathrm{P}$ is not inside circle $C$. Then

$$
J_{i i-1}=2 \int_{0}^{\varphi_{i i-1}} \mathrm{~d} \varphi \int_{f_{i i-1}{ }^{-}(\varphi)}^{R} \frac{h(\vartheta)}{\cos (\vartheta)} r \mathrm{~d} r .
$$

(2) $\quad R_{i 0}<R$ and $R<q_{i i-1}+a_{i i+1}$, i.e., the point of contact is inside circle $C$, and circle $C$ and ellipse $C_{i i-1}$ intersect. Then

$$
\begin{aligned}
J_{i i-1}= & 2 \int_{0}^{\varphi_{i i-1}} \mathrm{~d} \varphi \int_{f_{i i-1}{ }^{-}(\varphi)}^{R} \frac{h(\vartheta)}{\cos (\vartheta)} r \mathrm{~d} r \\
& +2 \int_{\varphi i i-1}^{\varphi_{i 0}} \mathrm{~d} \varphi \int_{f_{i i-1}{ }^{-}(\varphi)}^{f_{i i-1}{ }^{+}(\varphi)} \frac{h(\vartheta)}{\cos (\vartheta)} r \mathrm{~d} r .
\end{aligned}
$$

(3) $R_{i 0}<R$ and $R \geq q_{i i-1}+a_{i i-1}$, i.e., ellipse $C_{i i-1}$ is located inside circle $C$. Then

$$
J_{i i-1}=2 \int_{0}^{\varphi_{i 0}} \mathrm{~d} \varphi \int_{f_{i i+1}^{-}(\varphi)}^{f_{i i-1}{ }^{+}(\varphi)} \frac{h(\vartheta)}{\cos (\vartheta)} r \mathrm{~d} r .
$$

Integral $J_{i i+1}$ is calculated as

$$
J_{i i+1}=2 \int_{0}^{\varphi i i+1} \mathrm{~d} \varphi \int_{f_{i i+1}{ }^{-}(\varphi)}^{R} \frac{h(\vartheta)}{\cos (\vartheta)} r \mathrm{~d} r,
$$

and finally integral $J_{i i}$ is calculated as

$$
J_{i i}=2 \int_{0}^{\pi} \mathrm{d} \varphi \int_{0}^{R} \frac{h(\vartheta)}{\cos (\vartheta)} r \mathrm{~d} r-J_{i i-1}-J_{i i+1} .
$$

The sensitivity distribution $h(\vartheta)$ can be well approximated by a slightly modified Gaussian function

$$
h(\vartheta)=\exp \left[-m \sin ^{2}(\vartheta)\right] \cos (\vartheta),
$$

where the fitting coefficient $m$ adjusts the function to the individual curve of the microprobe under consideration.

From the relationship between the Cartesian and spherical coordinates for a unit sphere, $x=\sin (\vartheta) \cos (\psi)$ and $y=\sin (\vartheta) \sin (\psi)$, one can derive $\sin ^{2}(\vartheta)=x^{2}+y^{2}=$ $r^{2}$, which provides the following solution for the internal integral:

$$
\begin{aligned}
\int_{f_{1}(\varphi)}^{f_{2}(\varphi)} \exp \left(-m r^{2}\right) r \mathrm{~d} r & \\
& =\frac{1}{2 m}\left\{\exp \left[-m f_{1}^{2}(\varphi)\right]-\exp \left[-m f_{2}{ }^{2}(\varphi)\right]\right\} .
\end{aligned}
$$

Thus all the integrals $J_{i j}$ can be solved. On the grounds of symmetry,

$$
\begin{aligned}
J_{i i} & =J_{K-1-i, K-1-i}, \\
J_{i i-1} & =J_{K-1-i, K-i}, \\
J_{\mathrm{ii}+1} & =J_{K-1-i, K-i-2} .
\end{aligned}
$$

After determination of all the integrals $J_{i j}$, the $K$ unknown quantities $L_{i}(i=0,1, \ldots, K-1)$ can be found from $K$ equations

$$
\begin{aligned}
L_{i-1} J_{i i-1}+L_{i} J_{i i}+L_{i+1} J_{i i+1} & =M_{i}, \\
J_{0,-1}=J_{K-1, K} & =0 .
\end{aligned}
$$

\section{Example of Application}

In this subsection we apply the procedure described above to account for the nonuniform angular sensitivity of a microprobe that is used for three-dimensional equidistant radiance measurements in a coastal sediment with diatoms. A set of ten measurements as described in Fig. 2 has been carried out at each of four depths, $d=0,0.5,1.5,2 \mathrm{~mm}$, of a sediment sample immersed in water and irradiated with collimated light $(\lambda=650 \mathrm{~nm})$. Of course, the measurements assigned to depth $d=0$ are performed not on the external surface of the sample, where only the collimated component at $\theta_{0}$ would be measurable, but at a small distance below the surface. There is some uncertainty in determining this distance, first because of finite step size of the probe advancement and, second, because of the roughness of the surface, which in turn is caused by the finite size of the particles that constitute the sample. For samples such as those used in this example the uncertainty amounts to a few micrometers (see Ref. 7 for experimental details). The best fit of the measured (in water) bell-shaped angular sensitivity of the used microprobe with the function $h(\vartheta)=\cos (\vartheta) \exp (-m$ $\sin ^{2} \vartheta$ ) was achieved for $m=78.29$. In terms of standard nomenclature (see Section 1) this corresponds to an acceptance half-angle of $\theta_{a} \approx 8^{\circ}$.

For each of the four data sets Table 1 shows the measured quantities $M_{i}$ and uncorrected radiance $L_{i}{ }^{*}=M_{i} / S_{0}$, calculated under the assumption that Eq. (2) is valid, i.e., the angular sensitivity of the microprobe is presented by the dashed line in Fig. 3. These values are compared with the corrected radiance $L_{i}$ calculated from Eq. (3) using $m=78.29$ for $h(\vartheta)$. Table 1 also contains the weighting factors $W_{i}$ that are needed to calculate fluxes over the spherical bands and, finally, the fluence rate. The corrected and uncorrected values of fluence rates as well as the discrepancy between them are given for each depth. Also corrected versus uncorrected radiant fluxes over single spherical bands can be easily calculated. These data for depth $d=0.5 \mathrm{~mm}$ are shown in Fig. 9 .

The data presented in Table 1 and Fig. 9 show that in this case, by neglecting the nonuniformity of the 
Table 1. Comparison of Corrected and Uncorrected Radiance Values Obtained from Measurements at Different Depths of a Coastal Sediment with Diatoms $(\lambda=650 \mathrm{~nm})^{a}$

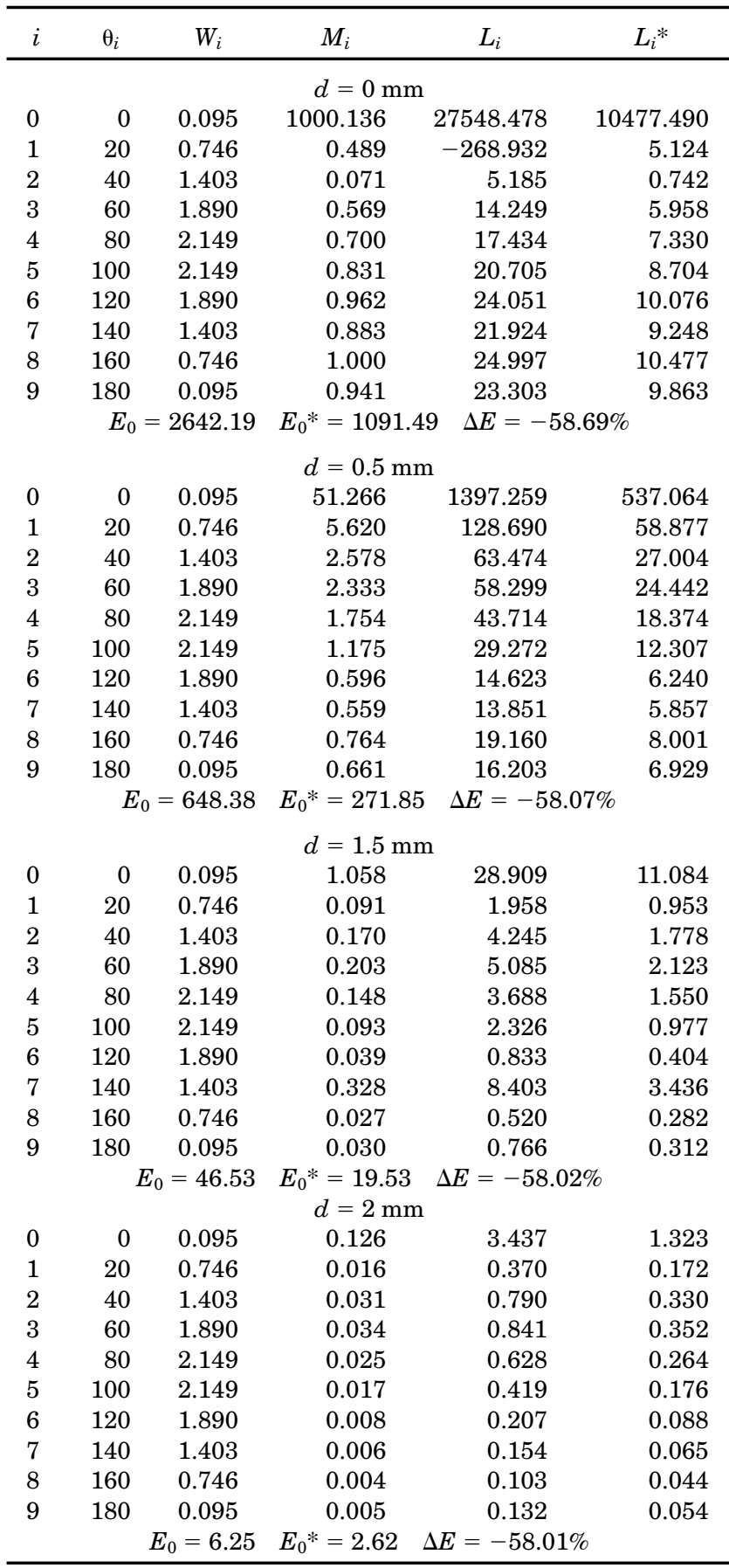

${ }^{a}$ Data are from Ref. $7 . \quad d(\mathrm{~mm})$ represents the depth of a set of measurements; $i$ is the number of a measurement within a set; $\theta_{i}$ (degrees) is the direction of the $i$ th measurement; $W_{i}$ is the area of the $i$ th spherical band, $W_{i}=2 \pi\left[\cos \left(\theta_{i}-\alpha\right)-\cos \left(\theta_{i}+\alpha\right)\right], 0<\theta_{i}<$ $\pi\left(\Sigma W_{i}=4 \pi\right) ; M_{i}$ (relative units normalized against direct illumination of the microprobe with the same light source) is the measured quantity; $L_{i}^{*}=M_{i} / S_{0}$ is the uncorrected radiance; $L_{i}$ is the corrected radiance calculated according to the above procedure. $E_{0}=\Sigma L_{i} W_{i}$ and $E_{0} *=\Sigma L_{i} * W_{i}$ represent the corrected and uncorrected fluence rates, respectively; $\Delta E=100\left(E_{0} *-E_{0}\right) / E_{0} \%$ represents the relative discrepancy between $E_{0} *$ and $E_{0}$.

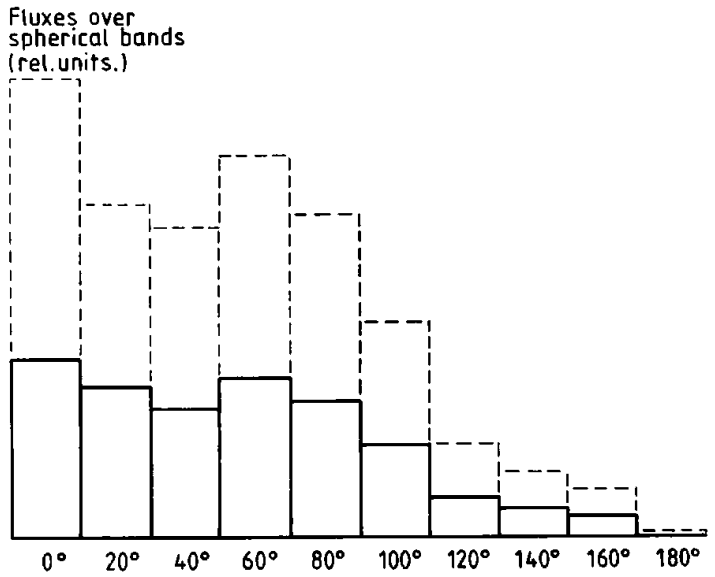

Fig. 9. Diagram of the corrected (dashed line) and uncorrected (solid line) radiant fluxes over single spherical bands. The bands are specified according to the directions of microprobe $\theta_{i}$ at successive measurements as presented in Fig. 2. The diagram presents measurements at depth $d=0.5 \mathrm{~mm}$. The corresponding radiances are also shown in Table 1 . The flux values are normalized against the incident flux.

probe angular sensitivity, we strongly underestimate the fluence rate. For the probe used in this example the relative discrepancy $\Delta E$ between the corrected $\left(E_{0}\right)$ and uncorrected $\left(E_{0} *\right)$ values of the fluence rate is approximately $60 \%$. If the same measured quantities $M_{i}$ are provided by a probe with larger acceptance angle $\theta=11^{\circ}$ [which corresponds to $m=45$ in $h(\vartheta)]$, the relative error in the scalar irradiance would be only approximately $25 \%$ (intermediate results of these calculations are not presented). A distinguished feature of the data in Table 1 is the negative value of corrected radiance at depth $d=0$. This phenomenon is expected under the given conditions of illumination and the geometry of the measurements. It is discussed in the next section.

\section{Discussion}

Generally there are three factors that affect the accuracy of the three-dimensional radiance measurements with radiance microprobes:

(i) The nonuniform angular sensitivity of the microprobe described above as function $h(\vartheta)$.

(ii) The zenithal spacing of single measurements specified above by angular interval $\alpha$ linked to the number of equidistant measurements $K$ through Eq. (1).

(iii) The angular radiance distribution solid of the incident radiation.

The effect of the microprobe sensitivity is qualitatively obvious. Large acceptance angles [i.e., broad distributions $h(\vartheta)]$ average too much information, especially in the direction of the maximum of the field radiance. They tend to broaden the shape of the distribution and at the same time tend to lower the apparent value of the peak radiance. Small 
acceptance angles [i.e., narrow distributions $h(\vartheta)]$ give more details about the radiance distribution but underestimate the fluence rate. In order to reduce these drawbacks the angular sensitivity of the microprobe was linked to the angular step between the measurements: $\theta_{a}=\alpha$. But even in this case the experimental data must be expected to contain an error of indefinite sign and magnitude.

The theory we present in this paper applies the independently obtained information about the angular sensitivity of the used microprobe to correct for this error that can be significant as shown in Section 2 . The correction procedure performed in our example for depth $d=0.5 \mathrm{~mm}$ (see Table 1 and Fig. 9) reveals rather moderate distortion of the radiance solid distribution. In contrast, the error in the value of the fluence rate is approximately $60 \%$. Similar conclusions can be drawn from the corrections at depths $d=1.5 \mathrm{~mm}$ and $d=2 \mathrm{~mm}$ (Table 1). In all these cases the lowered sensitivity of the probe toward the inclined components of radiation within the targeted band produces the dominating contribution to the instrumental error. The angular distribution of the radiation is in all these cases rather smooth (see Fig. 9). Therefore, the field radiance can be nicely recovered from the equidistant measurements because the angular size of the spherical bands, $2 \alpha$, is small enough to assume the constancy of the radiance over a spherical band. This assumption is not valid when the third factor that affects the accuracy of measurements, the angular distribution of radiation, has unfavorable properties. This is exactly the case in our example when the calculations for $d=0$ are performed. Here we have not only a strong underestimate of the fluence rate but also a significant distortion of the radiance solid distribution. The theory yields a negative value for $L_{1}$. This does not mean that the treatment is wrong. Moreover, the negative value of $L_{1}$ is to be expected for the following reasons. The collimated incident radiation is not spread uniformly over band number zero but concentrated in an extremely narrow solid angle. It does not affect the measured value $M_{1}$ in band number one. However, the theory treats the magnitude $M_{0}$ as being uniformly spread over band zero and accordingly accounts for the enormous (note that $M_{0}$ exceeds $M_{1}$ by 3 orders of magnitude!) but, in fact, nonexistent contribution of light from band number zero to $M_{1}$. This results in a large negative value for $L_{1}$.

Obviously, to account for strong peaks of the field radiance distribution, smaller zenithal steps between the measurements are required, at least in the neighborhood of such peaks. This means that an extension of the theory to nonequidistant measurements is desirable. Even more important is another consequence of introducing smaller zenithal steps. The angular size of spherical bands that correspond to single measurements may be in this case much smaller than the acceptance angle of the microprobe. Consequently a quantity $M_{i}$ will con- tain contributions not only from bands $i-1$ and $i+1$ but also from the more distant bands.

We can now summarize the discussion. The theory of equidistant three-dimensional measurements with the band size linked to the acceptance angle of the microprobe provides, for the first time, to our knowledge the quantitative processing of measured data. The errors that are due to the nonuniform angular sensitivity of a microprobe can now be estimated and, in general, a comparison of measurements performed with different microprobes is now possible. This theory is sufficient for smooth angular radiance distribution solids, for which the radiance can be considered constant within a spherical band linked to the acceptance angle of the microprobe. For the extreme cases of strongly peaked radiance distributions an extension of the theory to nonequidistant measurements, in which the band size is not linked to the acceptance angle of the microprobe, is desirable.

\section{Appendix A.}

We consider a Cartesian coordinate system $x P y$. An ellipse with the center at point $P_{1}(q, 0)$ and the half-axes $a, b$ with $(b \geq a)$ (Fig. 8) is described as

$$
\frac{(q-x)^{2}}{a^{2}}+\frac{y^{2}}{b^{2}}=1
$$

which yields

$$
y^{2}=b^{2}-\frac{b^{2}}{a^{2}}(q-x)^{2} .
$$

To obtain the ellipse equation in polar coordinates $r$ $=f(\varphi)$, first we express $r$ through $x$ :

$$
r^{2}=x^{2}+y^{2}=x^{2}+b^{2}-\frac{b^{2}}{a^{2}}(q-x)^{2} .
$$

Since $x=r \cos (\varphi)$ we obtain the relation between $r$ and $\varphi$ :

$$
r^{2}\left[1+\frac{c^{2}}{a^{2}} \cos ^{2}(\varphi)\right]-2 q \frac{b^{2}}{a^{2}} \cos (\varphi)+q^{2} \frac{b^{2}}{a^{2}}-b^{2}=0,
$$

with $c^{2}=b^{2}-a^{2}$, which results in

$$
r=\frac{q b^{2} \cos (\varphi) \pm a b\left[a^{2}-q^{2}+\left(q^{2}+c^{2}\right) \cos ^{2}(\varphi)\right]^{1 / 2}}{a^{2}+c^{2} \cos ^{2}(\varphi)} .
$$

When $a \geq q$, i.e., when point $\mathrm{P}$ is inside the ellipse, Eq. (A2) should be taken with the plus sign since, in this case,

$$
a b\left[a^{2}-q^{2}+\left(q^{2}+c^{2}\right) \cos ^{2}(\varphi)\right]^{1 / 2} \geq\left|q b^{2} \cos (\varphi)\right| .
$$


When $a<q$, i.e., when point $\mathrm{P}$ is outside the ellipse, a tangent to the ellipse drawn from point $\mathrm{P}$ always exists. The coordinates of the point of contact are designated as $R_{0}, \varphi_{0}$. Two values of $r$ correspond to one value of the coordinate $\varphi,|\cos (\varphi)|>\left|\cos \left(\varphi_{0}\right)\right|$, which is in accordance with the two signs in Eq. (A2). Point $\varphi_{0}$ is found when the discriminant in Eq. (A2) vanishes:

$$
\cos \left(\varphi_{0}\right)= \pm\left(\frac{q^{2}-a^{2}}{q^{2}+c^{2}}\right)^{1 / 2},
$$

whereas the sign of this expression is always the same as the sign of $q$ :

$$
R_{0}=r\left(\varphi_{0}\right)=\frac{\left[\left(q^{2}-a^{2}\right)\left(q^{2}+c^{2}\right)\right]^{1 / 2}}{|q|}
$$

The intersection point between the ellipse and circle $r=R$ has coordinates $\left(R, \varphi_{1}\right)$. The value of $\varphi_{1}$ is determined from Eq. (A1):

$$
\cos \left(\varphi_{1}\right)=\frac{1}{R c^{2}}\left\{q b^{2} \pm a\left[q^{2} b^{2}-c^{2}\left(R^{2}-b^{2}\right)\right]^{1 / 2}\right\}
$$

This research is supported by grant Fu 152/6-1 from Deutsche Forschungsgemeinschaft, the MaxPlanck Gesellschaft, and the Carlsberg foundation, Denmark (B. B. Jørgensen).

\section{References}

1. T. C. Vogelmann and L. O. Björn, "Measurements of light gradients and spectral regime in plant tissue with a fiber optic probe," Physiol. Plant. 60, 361-368 (1984).

2. T. C. Vogelmann, G. Martin, G. Chen, and D. Buttry, "Fiber optic microprobes and measurement of the light microenvironment within plant tissues," Adv. Bot. Res. 18, 255-295 (1991).

3. L. Fukshansky, N. Fukshansky-Kazarinova, and A. M. von Remisowsky, "Estimation of optical parameters in a living tissue by solving the inverse problem of the multiflux radiative transfer," Appl. Opt. 30, 3145-3153 (1991).

4. L. Lilge, T. Haw, and B. C. Wilson, "Miniature isotropic optical fibre probes for quantitative light dosimetry in tissues," Phys. Med. Biol. 38, 215-230 (1987).

5. B. B. Jørgensen and D. J. Des Marais, "A simple fiber-optic microprobe for high resolution light measurements: application in marine sediment," Limnol. Oceanogr. 31, 1376-1383 (1986).

6. M. Kühl and B. B. Jørgensen, "Spectral light measurements in microbenthic communities with a fiber-optic microprobe coupled to a sensitive diode array detector system," Limnol. Oceanogr. 37, 1813-1823 (1992).

7. M. Kühl and B. B. Jørgensen, "The light field of microbenthic communities: radiance distribution and microscale optics of sandy coastal sediments," Limnol. Oceanogr. 39, 1368-1398 (1994).

8. M. Kühl, C. Lassen, and B. B. Jørgensen, "Optical properties of microbial mats: light measurements with fiber-optic microprobes," in Microbial Mats: Structure, Development and Environmental Significance, L. J. Stal and P. Caumette, eds., NATO Advanced Studies Institute Series G (Springer-Verlag, Berlin, 1994), pp. 149-166.

9. J. M. Senior, Optical Fiber Communications: Principles and Practice (Prentice-Hall, New York, 1985).

10. P. S. Mudgett and L. W. Richards, "Multiple scattering calculations for technology," Appl. Opt. 10, 1485-1501 (1971). 